\title{
Characterization of Near Field Plasma Environment of a Hollow Cathode Assembly
}

\author{
Emily C. Fossum, ${ }^{*}$ Jason D. Sommerville, ${ }^{\dagger}$ and L. Brad King ${ }^{\ddagger}$ \\ Michigan Technological University, Houghton, MI 49931
}

\begin{abstract}
Cathode keeper erosion has been identified as a possible limit to thruster lifetimes. Therefore, it is important to characterize a cathode discharge at various operating conditions in order to identify potential erosion mechanisms. In this investigation the near field plasma environment of a hollow cathode assembly was examined under several operating conditions in the Isp Lab's Xenon Test Facility (XTF) at Michigan Tech. This examination focused on the region within $3 \mathrm{~cm}$ of the cathode keeper orifice. In this paper the results from six operating conditions are summarized giving values of plasma potential, electron temperature and number density. It was found that electron density did not vary significantly with changes in xenon flow rate and discharge current. It was also found that as flow rate increased, electron temperature decreased, resulting in a shorter Debye length and a flatter potential structure near the orifice; as discharge current increased, electron temperature also decreased.
\end{abstract}

\section{Nomenclature}

$A_{P} \quad=$ area of probe

$e \quad=$ elementary charge

$I_{D} \quad=$ discharge current

$I_{e o} \quad=$ electron saturation current

$I_{P} \quad=$ probe current

$k=$ Boltzmann's constant

$m_{c} \quad=$ cathode mass flow rate

$m_{e} \quad=$ mass of an electron

$n_{e} \quad=$ electron density

$n_{0} \quad=$ number density

$T_{e} \quad=$ electron temperature

$V_{D} \quad=$ discharge voltage

$V_{P} \quad=$ probe voltage

$\lambda_{D} \quad=$ Debye length

\section{Introduction}

TON thrusters are being designed to operate successfully for lifetimes exceeding 8,000 hours. Cathode erosion was Loriginally identified as a possible limit to thruster lifetime following a 2000 hour development wear-test ${ }^{1}$. In order to address this potential failure mechanism a keeper electrode was introduced, which dramatically reduced the cathode erosion. However not surprisingly, keeper erosion has emerged as a lifetime limiter and is a cause for concern in ion thruster design ${ }^{2}$. Although thicker orifice plates could potentially extend the lifetime of the keeper, this design modification may not be feasible in some cases. Therefore, understanding erosion mechanisms through cathode plume studies, in particular the potential structure downstream of the orifice, remain critical in thruster and cathode design.

\footnotetext{
* Ph.D. Candidate, Mechanical Engineering, 1019 R.L. Smith Bldg., 1400 Townsend Dr.

${ }^{\dagger}$ Ph.D. Candidate, Mechanical Engineering, 1018 R.L. Smith Bldg., 1400 Townsend Dr.

* Assistant Professor, Mechanical Engineering, 1014 R.L. Smith Bldg., 1400 Townsend Dr.
} 
Theoretical and experimental investigations have attempted to quantify low-energy ion sputtering $(<100 \mathrm{eV}))^{3,4}$ which is believed to be the cause of erosion in ion thrusters. The ion energy levels required for erosion and the origin of the ions is not well defined. Several hypotheses have attempted to explain the source of the impinging ions, the leading argument being a potential-hill phenomenon in the near field plasma ${ }^{5,6}$. Plasma oscillations have also been identified as a possible source for the impinging ions required for erosion ${ }^{6}$.

Several investigations have been conducted to characterize the plume downstream of a hollow cathode in order to gain insight on the nature of the impinging ions. Laser-induced fluorescence (LIF) has been used to examine Xe II velocities downstream of the anode that were consistent with a potential hill in the near field plasma discharge at high powers ${ }^{5,6}$. In the current investigation, Langmuir probes were used to characterize the near field plasma potential structure and electron temperatures within $3 \mathrm{~cm}$ of the keeper. This ongoing study attempts to support previous and future characterizations that use electrostatic probes, laser-induced fluorescence, and emission spectroscopy.

\section{Experimental Apparatus}

\section{A. Cathode}

The hollow cathode assembly used an orificed RMS hollow cathode, which was supplied to the Isp Lab by the NASA Glenn Research Center. The cathode created a low-temperature plasma $\left(T_{e}<5 \mathrm{eV}\right)$ by establishing a discharge between the hollow cathode chamber and a positive keeper electrode surrounding the cathode tip.

The cathode keeper had a $25 \mathrm{~mm}$ diameter with a $3 \mathrm{~mm}$ orifice. The cathode-keeper assembly was coaxially mounted inside a $100 \mathrm{~mm}$ (4 in.) diameter stainless steel cylinder, which served as an anode. Slits were cut down each side of the cylinder to allow for probe access. The anode was connected to a power supply that was referenced to the cathode and operated in current limited mode. The keeper power supply, also referenced to the cathode, was used for cathode ignition, and thereafter allowed to float. See Figure 1 for cathode setup and electrical schematic.
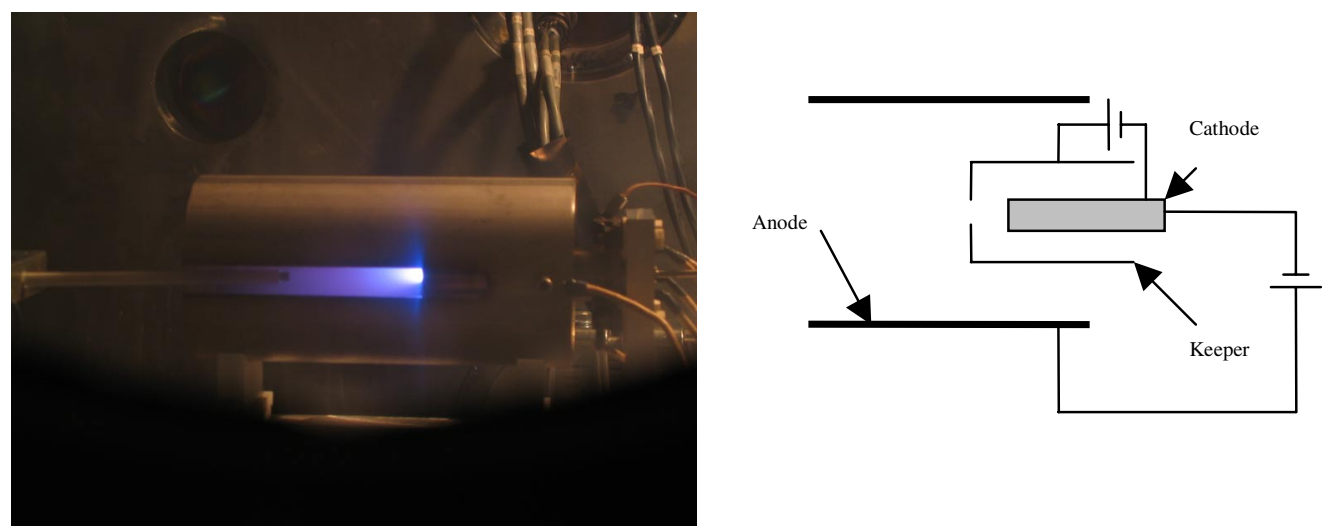

Figure 1: RMS hollow cathode assembly in operation (left) and electrical setup (right)

The cathode assembly was run at six different operating conditions using xenon, operating at three different flow rates and two different discharge currents. The background pressure varied from 6.2e-6 Torr at low flow rates to 1.6e-5 Torr for the highest flow rates. Upon visible inspection, it was determined that the cathode operated primarily in plume-mode, with the exception of the $7 \mathrm{sccm}, 10 \mathrm{~A}$ condition, which operated in spot mode. Table 1 summarizes the cathode operating conditions used in this investigation. 


\begin{tabular}{|c|c|c|c|}
\hline $\begin{array}{c}\text { Discharge Current }\left(\mathrm{I}_{\mathrm{D}}\right) \\
(\mathrm{A})\end{array}$ & $\begin{array}{c}\text { Discharge Voltage }\left(\mathrm{V}_{\mathrm{D}}\right) \\
(\mathrm{V})\end{array}$ & $\begin{array}{c}\text { Mass Flow Rate }\left(\mathrm{m}_{\mathrm{C}}\right) \\
(\mathrm{sccm})\end{array}$ & $\begin{array}{c}\text { Background Pressure } \\
(\text { Torr })\end{array}$ \\
\hline 5 & 30 & 3 & $6.4 \mathrm{e}-6$ \\
\hline 5 & 24 & 7 & $9.6 \mathrm{e}-6$ \\
\hline 5 & 22 & 15 & $1.6 \mathrm{e}-5$ \\
\hline 10 & 31 & 3 & $6.2 \mathrm{e}-6$ \\
\hline 10 & 25 & 7 & $1.0 \mathrm{e}-5$ \\
\hline 10 & 23 & 12 & $1.6 \mathrm{e}-5$ \\
\hline
\end{tabular}

Table 1: Hollow cathode operating conditions used in probe study of near field plasma of a hollow cathode.

\section{B. Testing Facility}

The Isp Lab's Xenon Test Facility was used for all testing in this investigation. The vacuum facility is a 2meter-diameter by 4-meter-long space simulation chamber. The chamber is evacuated to ultimate pressure using a 48-inch diameter liquid-nitrogen-assisted liquid-helium cryogenic vacuum pump, which can achieve a pumping speed of 60,000 liters per second on nitrogen. The cryopump is backed by a two-stage mechanical roughing pump capable of $200 \mathrm{~L} / \mathrm{s}$ pumping speed. The vacuum facility can achieve a pressure better than 1e-6 Torr. Xenon flow was controlled to the cathode using a dedicated propellant feed system incorporating a $20 \mathrm{sccm}$ full scale range mass flow controller with an accuracy of $\pm 1 \%$ of full scale $( \pm 0.20 \mathrm{sccm})$.

\section{Langmuir probe}

A cylindrical Langmuir probe was constructed with a $0.1 \mathrm{~mm}$ (5 mil) diameter tungsten filament with $1 \mathrm{~mm}$ exposed length and was insulated with $2 \mathrm{~mm}$ diameter alumina ceramic tubing. The probe was driven with a programmable source electrometer, which read the collected current while sweeping the probe from $-15 \mathrm{~V}$ to $50 \mathrm{~V}$ or $75 \mathrm{~V}$, depending on the particular plasma characteristics. The source meter was controlled by a data acquisition program written in $\mathrm{G}$.

The probes were mounted on a computer-controlled motion table capable of rotation and translational motion along two axes. The positioning system mapped out a 2 $\mathrm{mm}$ resolution grid where probe sweeps were taken in the near field of the hollow cathode. Figure 2 shows a schematic of the probe setup.

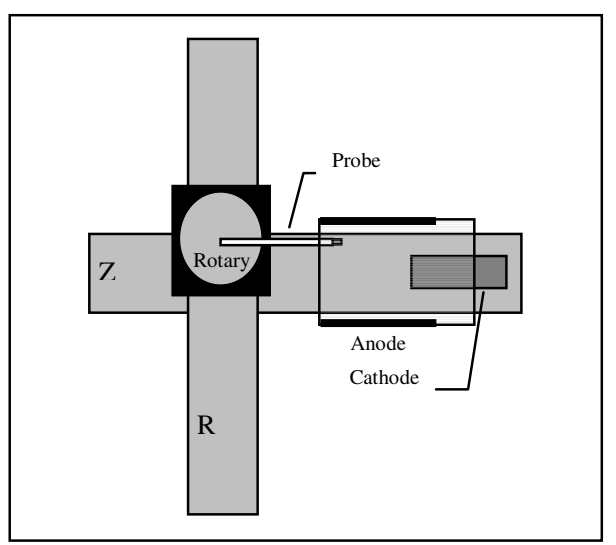

Figure 2: Setup of the probe positioning system for near field plasma environment characterization showing the Langmuir probe mounted on the positioning table. (not drawn to scale)

\section{Automated Analysis}

In order to expedite the analysis of a large number of Langmuir traces, an automated algorithm for their processing has been developed. The algorithm follows standard Langmuir analysis procedures ${ }^{7}$ and is implemented in $\mathrm{G}$ for easy integration into data acquisition programs. The algorithm extracts four parameters from the traces: floating potential, plasma potential, electron temperature, and an electron density estimate. It executes as follows:

1) Find floating potential

2) Find plasma potential by interrogating the first derivative

3) Find electron temperature via regression

4) Optionally revise plasma potential by an intersection method

5) Estimate electron density

Floating potential is first found by scanning the trace for a zero current crossing, starting from the negative voltages. The potential is interpolated from a line between the positive and negative data points on either side of zero current. 
The first method used to find plasma potential was the inflection point method, i.e. finding the peak in the first derivative that occurs at probe potentials positive of floating potential. A numeric derivative of the data is taken. If the data is noisy, a smoothing algorithm may also be applied. The first derivative was scanned and its peaks recorded. To avoid noise-induced peaks, only peaks above a certain, specifiable voltage width were recorded. Ideally there would be a single peak near floating potential. However noisy data may lead to multiple peaks and thus intelligence was applied when selecting a peak. Each peak was weighted based on how close it was to floating potential and on the total magnitude of the peak. The two weights were multiplied and the highest peak was selected as plasma potential. The remaining peak weights were subtracted from the selected peak's weight and the differences were multiplied. This result was subtracted from one to yield a validity parameter between 0 and 1 that describes the confidence in the selected peak. A characteristic trace is shown in Figure 3 that graphically shows the steps taken by the algorithm to determine plasma potential.

The algorithm also uses an intersection method as a second means to determine plasma potential. The details of the intersection method are also shown graphically in Figure 3. The algorithm uses a linear curve fit at the two portions of the curve shown in order to determine an intersection point, which it then calls plasma potential.

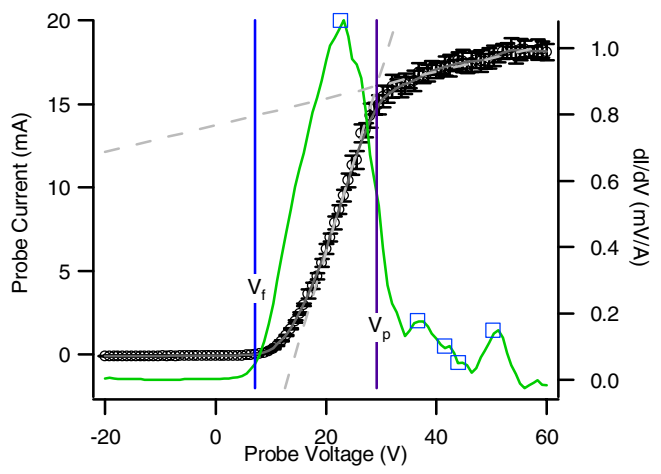

$$
\begin{array}{|cl|}
\hline \circ & \text { Probe Current } \\
\hline & \text { First Derivative } \\
\square & \text { Derivative Peaks } \\
\hline & \text { Floating Potential } \\
\hline & \text { Plasma Potential }
\end{array}
$$

Figure 2: Characteristic trace graphically outlining the steps taken by the algorithm to find plasma potential. When the inflection method was used, the voltage where the largest peak occurred was used as plasma potential. When the intersection method was used the plasma potential was selected at the intersection of the two dashed lines shown.

Once an estimate for plasma potential was found, the algorithm extracted the samples between floating and plasma potentials and took the natural log of the current. Linear regression was performed on this data to yield the electron temperature according to:

$$
\frac{d \ln I_{P}}{d V_{p}}=\frac{e}{k T_{e}}
$$

where $I$ is the probe current, $V$ is the probe bias, $e$ is the elementary charge, $k$ is Boltzmann's constant and $T_{e}$ is electron temperature.

Electron saturation is required in order to calculate electron density. Where electron saturation was achieved, electron density was calculated according to:

$$
n_{0}=\frac{I_{e 0}}{A e} \sqrt{\frac{2 \pi m_{e}}{k T_{e}}}
$$

\section{E. Sheath Effects}

The density estimate is most important for verifying the validity of the thin sheath analysis used by the algorithm. The Langmuir probe drew currents on the order of $1.0 \mathrm{~mA}$ at electron saturation. The probe's exposed area was approximately $.388 \mathrm{~mm}^{2}$. The electron temperatures were on the order of $1 \mathrm{eV}$. According to equation (2) this would yield an electron density on the order of $10^{19} \mathrm{~cm}^{-3}$. Using this estimate for density, the Debye length was calculated by: 


$$
\lambda_{D}=\sqrt{\frac{\varepsilon_{0} k T_{e}}{n_{e} e^{2}}}
$$

and is on the order of $\sim 2 \mu \mathrm{m}$. Since sheath size is typically several Debye lengths, the thin sheath assumption requires the Debye length to be sufficiently less than the probe characteristic length, which in this case would be the diameter of $0.12 \mathrm{~mm}$. The Debye length is less than 2 percent of the probe diameter; therefore, thin sheath analysis is warranted.

\section{Results}

\section{A. Plasma Potential and Electron Temperature}

The plasma potential and electron temperatures were found within $3 \mathrm{~cm}$ of the cathode keeper orifice using the Langmuir probe method and automated analysis previously described. Because the $\mathrm{V}_{\mathrm{p}}$ values found using the inflection point method contained a large amount of noise, the plasma potential values presented were found using the intersection method and thus can be considered an upper bound on the true value (see Figure 3). Contour plots are shown in Figure 3 and Figure 4 at three different flow rates for discharge currents of 5 Amps and 10 Amps, respectively. Electron temperatures fell within the range 0.2 to $3.5 \mathrm{eV}$; plasma potential ranged from $0 \mathrm{~V}$ to $30 \mathrm{~V}$. 
Xenon Flow Rate $\left(m_{c}\right)$ : 3 sccm; Discharge Current $\left(I_{D}\right): 5$ Amps; Discharge Voltage $\left(V_{D}\right)$ : $30 \mathrm{~V}$
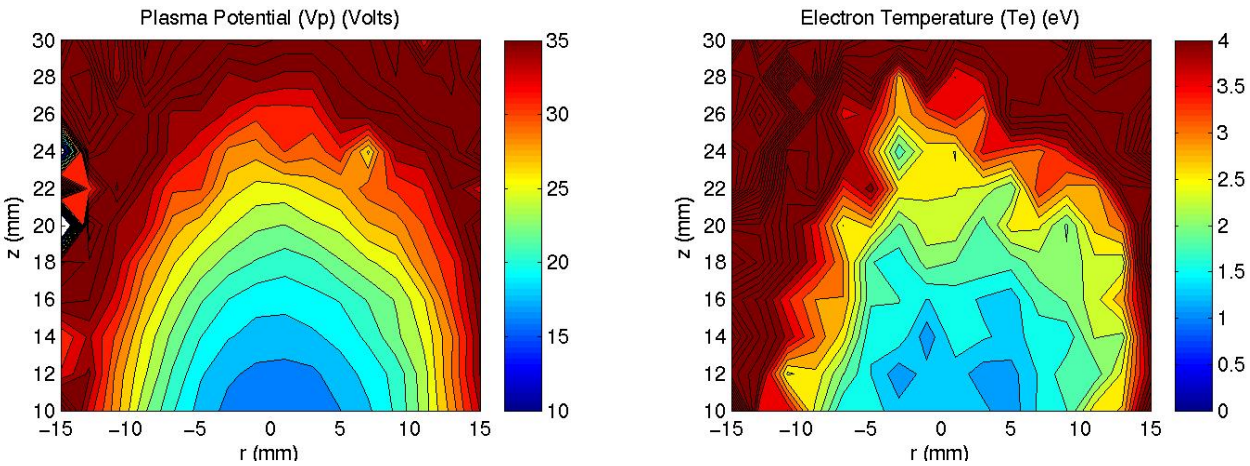

Xenon Flow Rate $\left(m_{c}\right): 7$ sccm; Discharge Current $\left(I_{D}\right): 5$ Amps; Discharge Voltage $\left(V_{D}\right): 24 V$
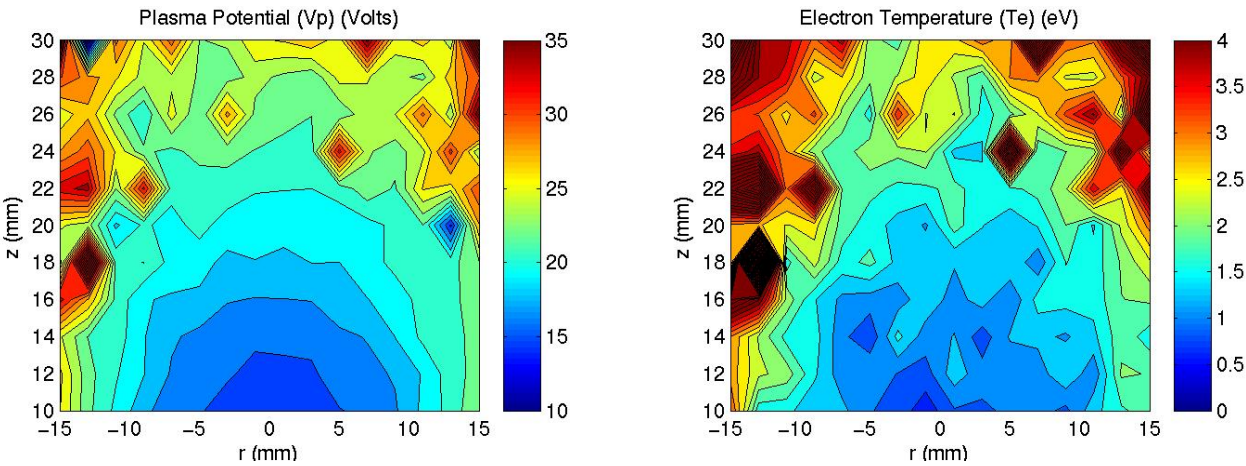

Xenon Flow Rate $\left(\mathrm{m}_{\mathrm{c}}\right)$ : $15 \mathrm{sccm}$; Discharge Current $\left(\mathrm{I}_{\mathrm{D}}\right)$ : 5 Amps; Discharge Voltage $\left(\mathrm{V}_{\mathrm{D}}\right)$ : $22 \mathrm{~V}$
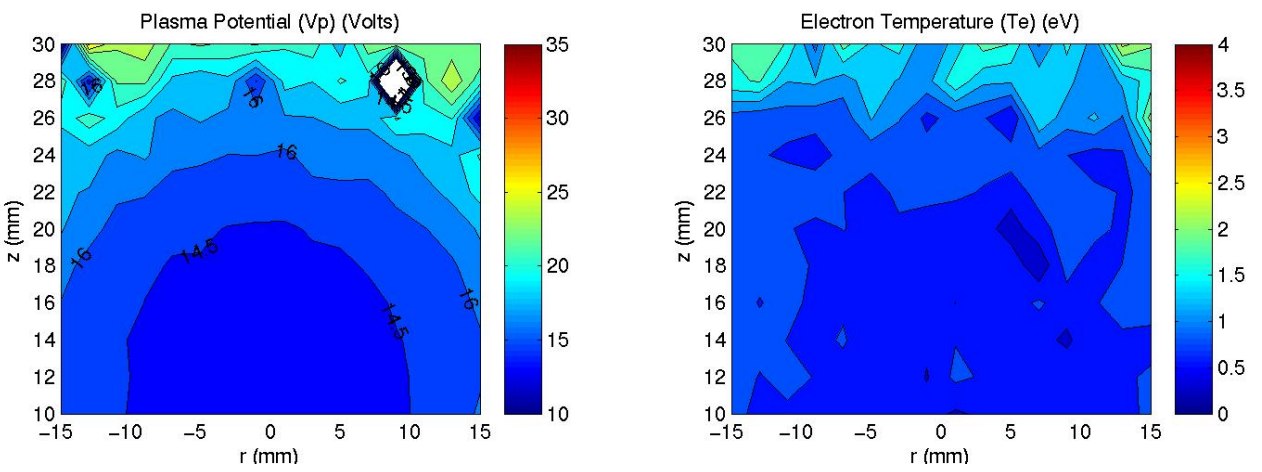

Figure 3: Plasma potential and electron temperature contour maps at a discharge current of 5 Amps for flow rates of $3 \mathrm{sccm}, 7 \mathrm{sccm}$, and $15 \mathrm{sccm}$. Discharge voltage was 30V, 24V and 22 V, respectively. 
Xenon Flow Rate $\left(m_{c}\right): 3 \mathrm{sccm}$; Discharge Current $\left(\mathrm{I}_{\mathrm{D}}\right)$ : 10 Amps; Discharge Voltage $\left(\mathrm{V}_{\mathrm{D}}\right)$ : $31 \mathrm{~V}$
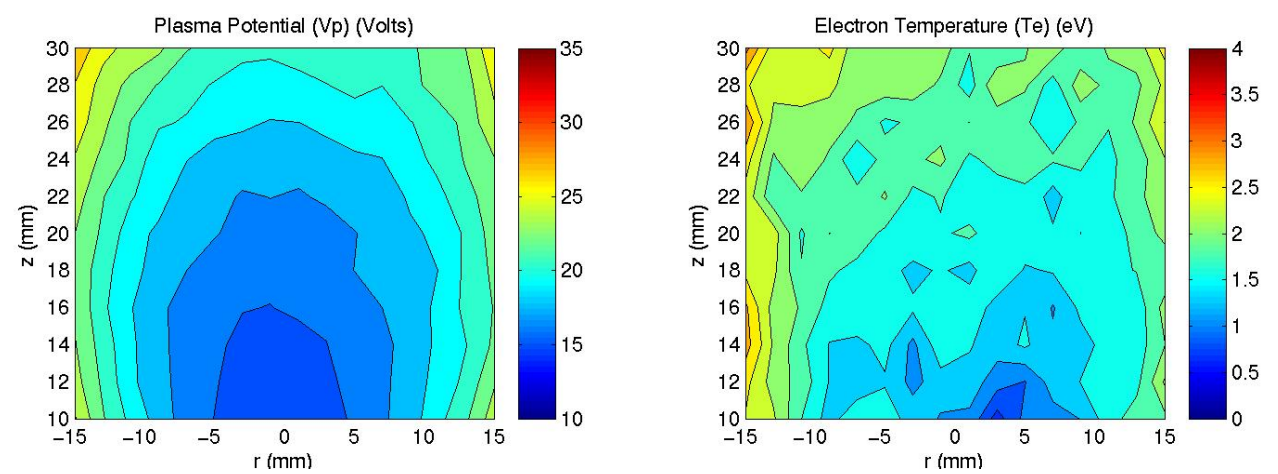

Xenon Flow Rate $\left(m_{c}\right): 7$ sccm; Discharge Current $\left(I_{D}\right): 10$ Amps; Discharge Voltage $\left(V_{D}\right): 25 \mathrm{~V}$
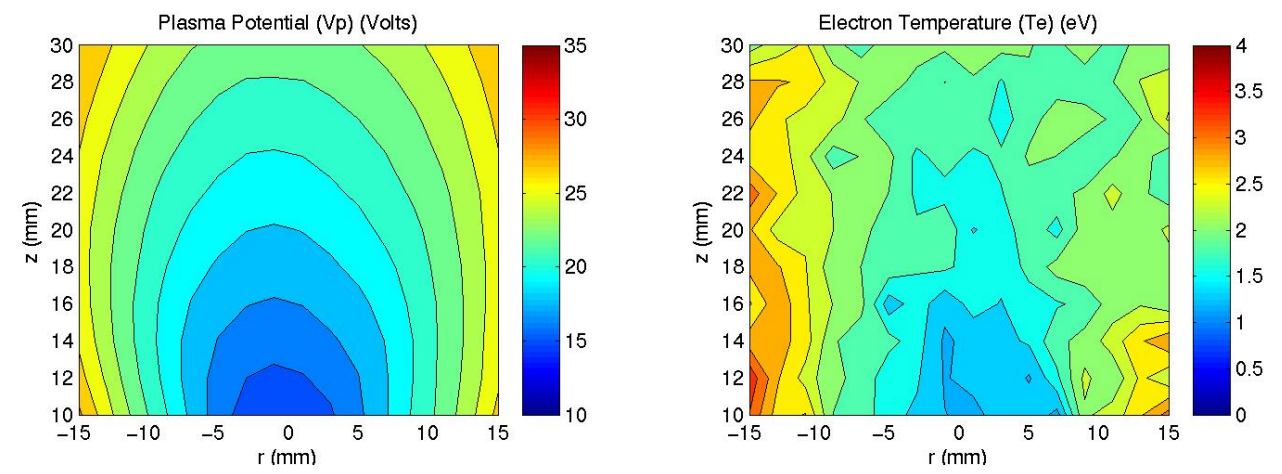

Xenon Flow Rate $\left(m_{c}\right): 12 \mathrm{sccm}$; Discharge Current $\left(\mathrm{l}_{\mathrm{D}}\right)$ : 10 Amps; Discharge Voltage $\left(\mathrm{V}_{\mathrm{D}}\right)$ : $23 \mathrm{~V}$
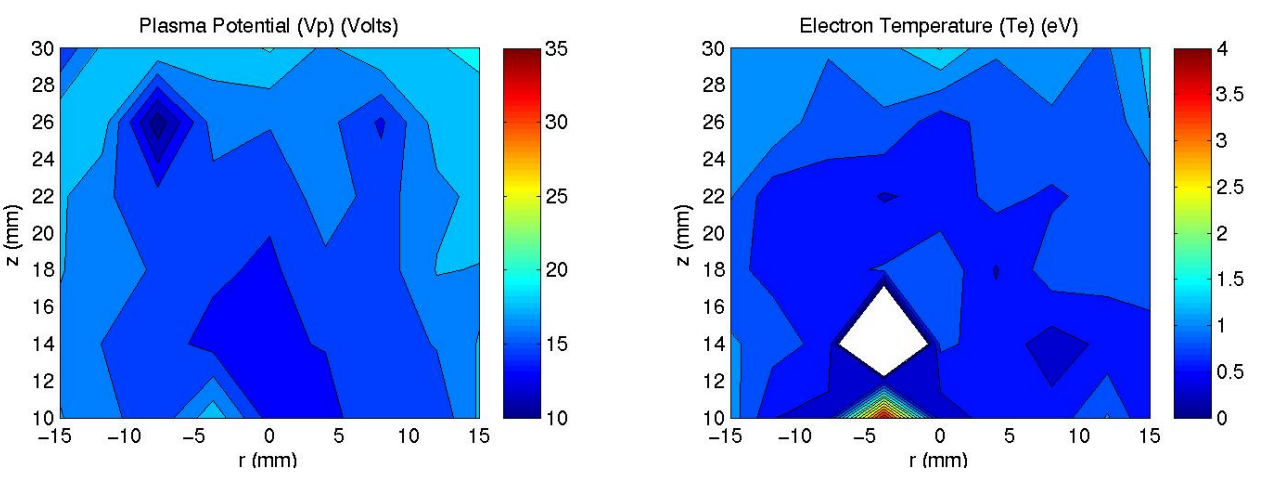

Figure 4: Plasma potential and electron temperature contour maps at a discharge current of 10 Amps for flow rates of $3 \mathrm{sccm}, 7 \mathrm{sccm}$, and $12 \mathrm{sccm}$. Discharge voltage was $31 \mathrm{~V}, 25 \mathrm{~V}$, and $23 \mathrm{~V}$, respectively. 
Cross sections of plasma potential are helpful for interpreting the data shown in Figure 4 and Figure 5. Figure 5 shows these cross sections taken at $14 \mathrm{~mm}$ downstream of the cathode for three flow rates at a discharge current of 5 Amps and 10 Amps. Uncertainty measures were found from the validity parameter plots described in the automated analysis algorithm.
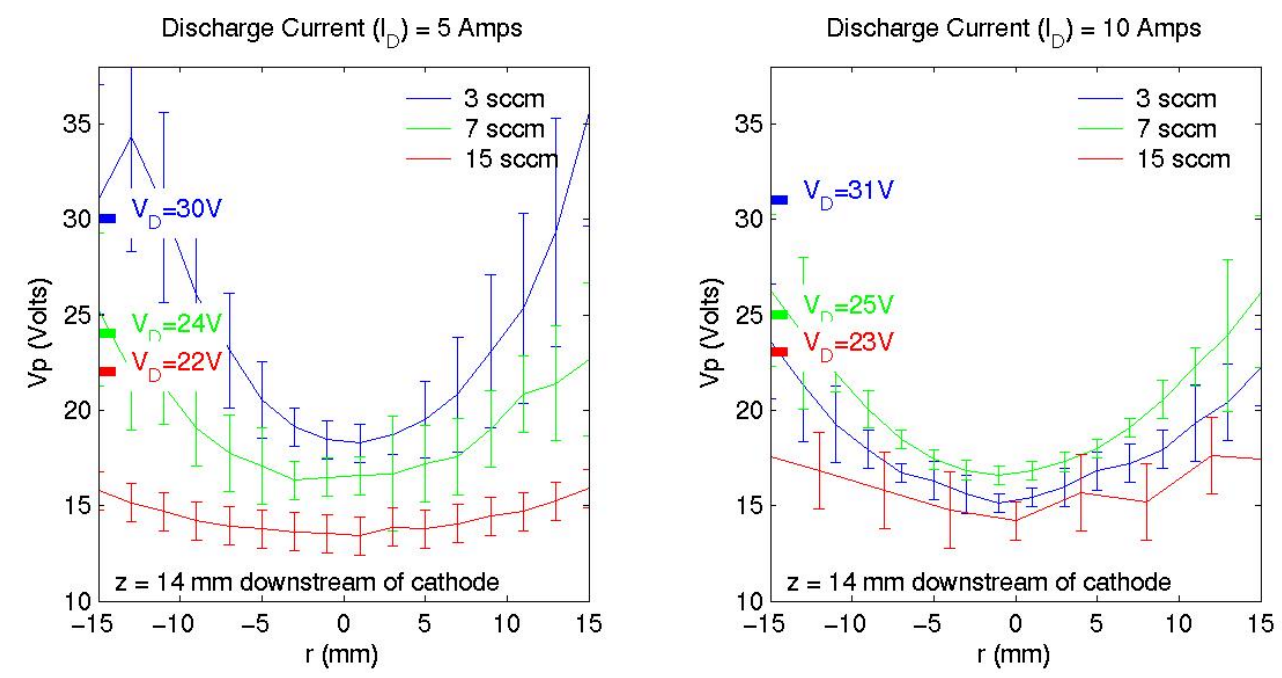

Figure 5: Cross section of plasma potential for the six cathode operating conditions, 5 Amp discharge current (left) and 10 Amp discharge current (right), at $14 \mathrm{~mm}$ downstream of the cathode keeper orifice. The cathode operated in plume mode for all conditions except at $7 \mathrm{sccm}$ flow rate, 10 Amp discharge current, at which point it operated in spot mode.

\section{B. Density}

Electron density was determined by the Langmuir probe method for the grid points that achieved clear electron saturation. Densities from the center of the cathode were found along the centerline of the cathode at $r=0$ for the six different operating conditions and are plotted in Figure 6 versus distance from the keeper orifice. Densities are on the order of $10^{19}$ and are found using the thin sheath assumption. At large values of $r$, probe traces did not conclusively saturate, hence density calculations were not possible.
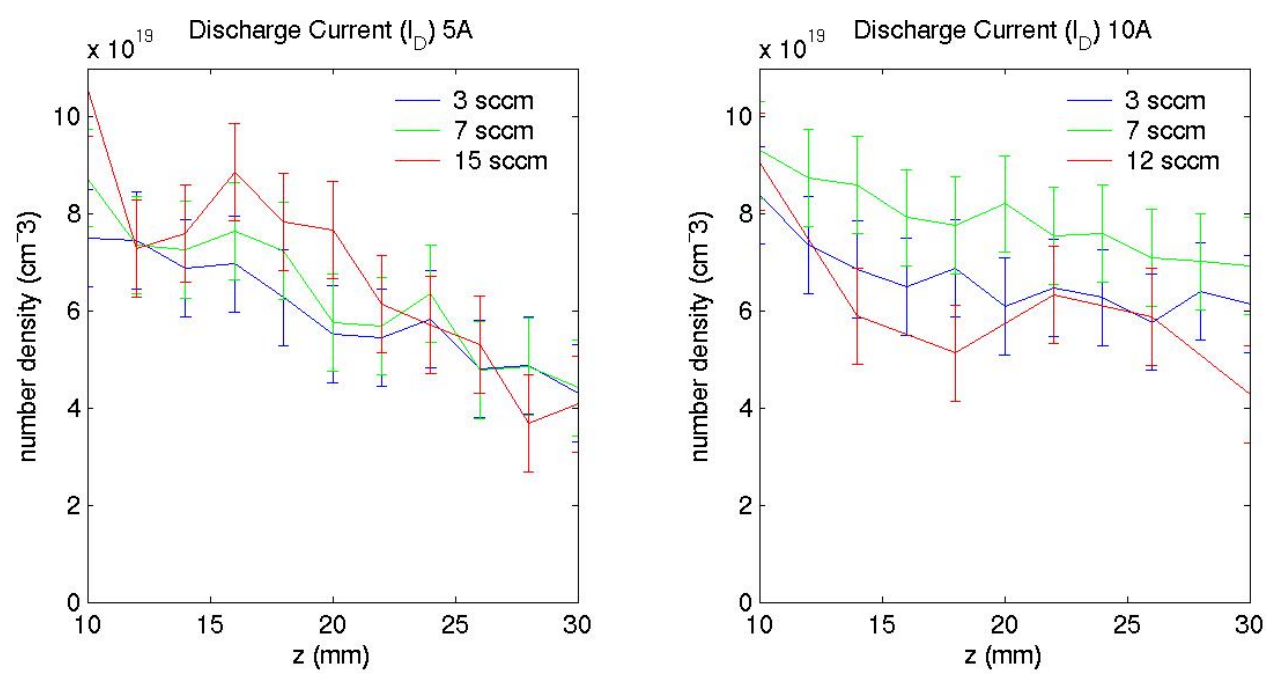

Figure 6: Electron densities along the center axis of the cathode $(r=0)$ are plotted versus distance from the cathode keeper orifice. The cathode operated in plume mode for all conditions except at $7 \mathrm{sccm}$ flow rate, 10 Amp discharge current, at which point it operated in spot mode. 


\section{Discussion}

The data collected in this investigation was used to characterize the near field hollow cathode plume at several operating conditions. Three flow rates were chosen at each of two discharge currents in order to differentiate behaviors based on xenon flow rate and discharge current independently.

\section{A. Electron Density}

Given the uncertainty in density calculations the electron density did not vary significantly with either increased current or flow rate. The electron density decreased with increased distance from the cathode orifice, and it appeared to decrease more rapidly for the $5 \mathrm{Amp}$ discharge current than for the $10 \mathrm{Amp}$ discharge current.

\section{B. Electron Temperature}

When discharge current is held constant electron temperatures decrease with increasing xenon flow rates (Figure 3 and Figure 4). Because the electron densities are on the same order for all flow rates (Figure 6), the apparent drop in electron temperature as flow rates are increased is believed to be due to a higher number of collisions with neutral xenon atoms.

When the flow rates are held constant, an increase in discharge current corresponds to lower electron temperatures. For example Figure 3 and Figure 4 show that for $7 \mathrm{sccm}$, plasma electrons achieved temperatures of $\sim 2.5 \mathrm{eV}$ for a $5 \mathrm{Amp}$ discharge current and $\sim 1.8 \mathrm{eV}$ for $10 \mathrm{Amp}$ discharge current at about $25 \mathrm{~mm}$ downstream of the keeper. The square of the Debye length scales inversely with electron density. Because electron density is maintained at 10 Amps and falls off more quickly for 5 Amps (Figure 6), it can be concluded that the electron temperature correlates with the Debye length. For higher density in the 10 Amp case the Debye length is lower and thus Debye shielding is enhanced. The potential structure near the cathode will be "flatter" because of this, corresponding to lower energy electrons near the cathode

\section{Plasma Potential}

Since the Debye length scales directly with $T_{e}^{1 / 2}$ and $n_{e}^{-1 / 2}$, the Debye length would be reduced if electron temperature decreased while electron density was held constant. A shorter Debye length corresponds to enhanced Debye shielding. This enhanced Debye shielding is apparent when comparing the potential cross sections in Figure 6. The higher flow rates correspond to lower electron temperatures, electron densities are essentially constant for constant discharge current so it would be expected that Debye length would decrease with increased flow rates. It is apparent that in the $3 \mathrm{sccm}, 5 \mathrm{Amp}$ condition, which indicates that the $15 \mathrm{sccm}, 5 \mathrm{Amp}$ case shows evidence of greater Debye shielding. This trend is apparent in all operating conditions, with the exception of the $7 \mathrm{sccm}, 10$ Amp case.

\section{Conclusions}

It was found that electron density did not vary significantly with changes in xenon flow rate. The electron density was on the same order for both discharge currents, however the density fell off more rapidly with distance from the cathode at a lower discharge current. It was found that electron temperature decreased with increased flow rate, likely due to collisions with slow neutrals. The decreased electron temperature resulted in a shorter Debye length, which corresponded to a flatter plasma potential structure near the orifice for higher flow rates. The electron temperature near the cathode also decreased with increased discharge current. Because the electron density was maintained with distance from the cathode at higher discharge currents, the Debye length was shorter and thus had a flatter potential structure in this region corresponding to lower energy electrons

The potential-hill phenomenon was not observed in this characterization, possibly because the measurements obtained here were taken at distances greater than $1 \mathrm{~cm}$ from the orifice. Other studies have shown evidence of potential hill between 1 and $11 \mathrm{~mm}$ downstream; therefore, future tests are planned to characterize the region of the cathode inside of $11 \mathrm{~mm}$.

\section{Acknowledgments}

This work was made possible by the continuing support of NASA Glenn Research Center by means of a research assistantship through Michigan Tech and through personnel at GRC, in particular Don Jaworski (grant monitor) and George Williams, that have been mentors throughout this project. 


\section{References}

${ }^{1}$ Patterson, M. J., et al "2.3 kW Ion Thruster Wear Test," AIAA-95-2516, $31^{\text {st }}$ Joint Propulsion Conference, July, 1995.

${ }^{2}$ Domonkos, M. T., Foster, J. E. and Patterson, M. J., "Investigation of Keeper Erosion in the NSTAR Ion Thruster," IEPC-01-308, 27 International Electric Propulsion Conference, October, 2001.

${ }^{3}$ Duchemin, O. B., et al, “A Review of Low Energy Sputtering Theory and Experiments," IEPC-97-068, 25 ${ }^{\text {th }}$ International Electric Propulsion Conference, August 1997.

${ }^{4}$ Nakles, M. R., et al, "Experimental and Modeling Studies of Low Energy Ion Sputtering in Ion Thrusters," AIAA-2003-5160, 39 ${ }^{\text {th }}$ Joint Propulsion Conference, July, 2003

${ }^{5}$ Williams, G. J., Jr., "The Use of Laser-Induced Fluorescence to Characterize Discharge Cathode Erosion in a $30 \mathrm{~cm}$ Ring-Cusp Ion Thruster,” Ph.D. Dissertation, University of Michigan, Ann Arbor, MI, 2000.

${ }^{6}$ Williams, G. J., Jr., Smith, T. B., Patrick, T. A., and Gallimore, A. D., "Characterization of the FMT-2 Discharge Cathode Plume," IEPC-99-104, $26^{\text {th }}$ International Electric Propulsion Conference, October 1999.

${ }^{7}$ Schott, L., "Electrical Probes," in Lochte-Holtgreven, W., Plasma Diagnostics, American Institute of Physics Press, Ch. 11, pp. 668-731, 1995. 